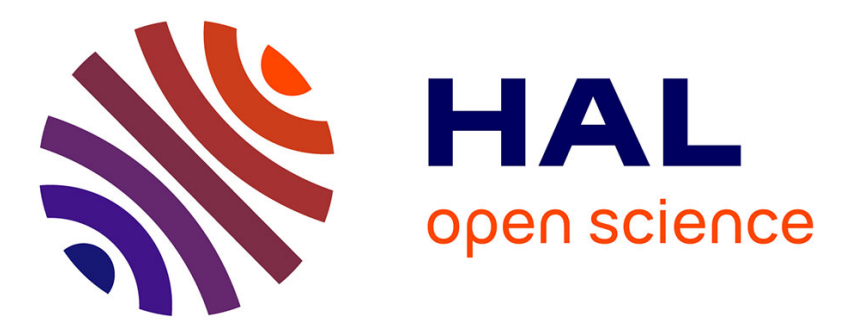

\title{
Axiparabola: a long-focal-depth, high-resolution mirror for broadband high-intensity lasers
}

Slava Smartsev, Clément Caizergues, Kosta Oubrerie, Julien Gautier, Jean-Philippe Goddet, Amar Tafzi, Kim Ta Phuoc, Victor Malka, Cédric Thaury

\section{To cite this version:}

Slava Smartsev, Clément Caizergues, Kosta Oubrerie, Julien Gautier, Jean-Philippe Goddet, et al.. Axiparabola: a long-focal-depth, high-resolution mirror for broadband high-intensity lasers. Optics Letters, 2019, 44 (14), pp.3414. 10.1364/OL.44.003414 • hal-02348979

\section{HAL Id: hal-02348979 https://hal.science/hal-02348979}

Submitted on 6 Nov 2019

HAL is a multi-disciplinary open access archive for the deposit and dissemination of scientific research documents, whether they are published or not. The documents may come from teaching and research institutions in France or abroad, or from public or private research centers.
L'archive ouverte pluridisciplinaire HAL, est destinée au dépôt et à la diffusion de documents scientifiques de niveau recherche, publiés ou non, émanant des établissements d'enseignement et de recherche français ou étrangers, des laboratoires publics ou privés. 


\title{
Axiparabola: a long-focal-depth, high-resolution mirror for broadband high-intensity lasers
}

\author{
Slava Smartsev, ${ }^{1,2, *}$ Clément Caizergues, ${ }^{2}$ Kosta Oubrerie, ${ }^{2}$ Julien Gautier, ${ }^{2}$ Jean-Philippe Goddet, ${ }^{2}$ \\ Amar Tafzi, ${ }^{2}$ Kim Ta Phuoc, ${ }^{2}$ Victor Malka, ${ }^{1,2}$ and Cédric Thaury ${ }^{2}$ \\ ${ }^{1}$ Department of Physics of Complex Systems, Weizmann Institute of Science, Rehovot 7610001, Israel \\ ${ }^{2}$ Laboratoire d'Optique Appliquée, Ecole Polytechnique, ENSTA, CNRS, Université Paris Saclay, Palaiseau, France \\ ${ }^{*}$ Corresponding author: slava.smartsev@weizmann.ac.il
}

Received 22 April 2019; revised 14 May 2019; accepted 7 June 2019; posted 10 June 2019 (Doc. ID 365351); published 3 July 2019

\begin{abstract}
Diffraction puts a fundamental limit on the distance over which a light beam can remain focused. For about 30 years, several techniques to overcome this limit have been demonstrated. Here, we propose a reflective optics, namely, the axiparabola, that allows to extend the production of "diffraction-free" beams to high-peak-power and broadband laser pulses. We first describe the properties of this aspheric optics. We then analyze and compare its performances in numerical simulations and in experiments. Finally, we use it to produce a plasma waveguide that can guide an intense laser pulse over 10 millimeters. @ 2019 Optical Society of America
\end{abstract}

https://doi.org/10.1364/OL.44.003414

Provided under the terms of the OSA Open Access Publishing Agreement

Optical elements with long focal depths and narrow lateral widths are required for a variety of applications, such as material processing [1], optical guiding of microscopic particles [2], optical coherence tomography [3], and formation of plasma waveguides [4]. Conventional optical elements, such as spherical lenses and parabolic mirrors, cannot achieve the two goals simultaneously, since a long focal depth requires a low numerical aperture and a high lateral resolution requires a large numerical aperture. These features are, however, naturally obtained with axicons [5], which are optical elements that produce approximations of Bessel beams [6]. Unfortunately, the focal depth and the lateral resolution cannot be chosen independently (assuming a fixed input beam size). In addition, an axicon generates a focal line with a non-constant axial intensity and with a low light efficiency. To overcome these drawbacks, various axilenses and generalized axicons have been suggested as diffractive optical elements that generate a constant axial intensity distribution and a narrow lateral width within an arbitrary long focal range [7-12]. However, these elements are not well suited for broadband spectra and high-intensity lasers, since they are wavelength dependent and have a low damage threshold.

In this Letter, we present the first reflective implementation of an axilens, which has negligible dependence on wavelength and high damage threshold as compared to refractive or diffractive alternatives. This reflective element essentially combines the long focal depth of an axicon and the high-energy concentration of a parabolic mirror; therefore, in analogy to an "axilens," we will further refer to it as an "axiparabola." When used with high-power ultra-short laser pulses, an axiparabola generates a long focal line with high peak intensity. This property makes the axiparabola a promising tool for generating low-density plasma channels, which are necessary for achieving high-energy electrons in laser plasma accelerators $[4,13,14]$. In addition, the axiparabola may be useful for other applications, including ultrashort laser pulse compression [15], extending the length of plasma filaments generated by intense laser pulses [16], $\mathrm{THz}$ generation [17], and lightning control $[18,19]$.

We designed and manufactured an axiparabola with an offaxis angle of $12^{\circ}$, a nominal focal length of $185 \mathrm{~mm}$, and a $20 \mathrm{~mm}$ focal depth. This axiparabola was tested experimentally and compared to simulation results. Last, we demonstrated the guiding of a terawatt (TW) laser pulse in a pre-formed plasma channel. The last was generated by focusing an additional TW laser by the axiparabola into a low-density hydrogen gas.

We start from a symmetrical on-axis axiparabola design. Consider a radially symmetric aspheric mirror with a sag function defined as $s(r)$, while $r$ is the radial coordinate, and $z$ is the main optical axis [Fig. 1(a)]. Using geometrical optics laws, it follows that rays parallel to the optical axis at coordinate $r$ will impinge on the surface at $z=s(r)$, then reflect from it and cross the main axis at $z=f(r)$, fulfilling the equation

$$
s(r)+\frac{r}{2}\left(\frac{1}{D(r)}-D(r)\right)=f(r),
$$

where $D(r) \equiv d s / d r$ is the sag derivative with respect to the radial coordinate. Solving for $D(r)$ and using the positive solution provides the differential equation for the sag function:

$$
\frac{s(r)-f(r)}{r}+\frac{1}{r} \sqrt{(f(r)-s(r))^{2}+r^{2}}=D(r) .
$$

Solving this equation for $f(r)=f_{0}$ leads to the well-known parabola case, with the sag function $s(r)=r^{2} / 4 f_{0}$.

In general, $f(r)$ could be chosen to lead to nonconstant intensity of the focal line. Here, we are interested in a constant case, which is obtained (assuming a uniform circular illumination) for 
(a)

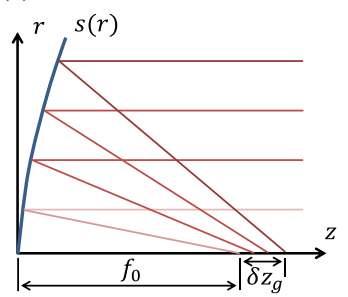

(b)

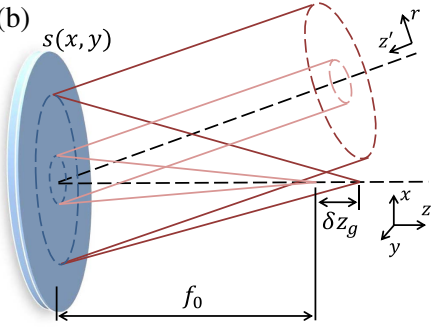

Fig. 1. Geometrical parameters and schematic presentation of rays focused by an axiparabola with positive $\delta z_{g}$. (a) On-axis case: incident rays that are far from the optical axis $z$ are focused after the nominal focus $f_{0}$ on the same axis; (b) off-axis case: incident rays that are far from the optical axis $z^{\prime}$ are focused after the nominal focus $f_{0}$ on the axis $z$. The off-axis angle is defined between axes $z$ and $z^{\prime}$.

$$
f(r)=f_{0}+\delta z_{g}(r / R)^{2},
$$

where $R$ is the radius of the incoming beam. Rays initially on the optical axis $(r=0)$ are focused at $f_{0}$, while rays initially on the aperture's edge $(r=R)$ are focused at $z=f_{0}+\delta z_{g}$; therefore, $\left|\delta z_{g}\right|$ is the focal depth length. Note that the sign of $\delta z_{g}$ can be either positive or negative. In the case of $\delta z_{g}>0$, which is illustrated in Fig. 1, rays with small $r$ are focused closer to the optics than rays with large $r$, while the opposite occurs for $\delta z_{g}<0$. The solution for the sag $s(r)$ can be derived as a power series of the radial coordinate $r$ :

$$
s(r)=\frac{1}{4 f_{0}} r^{2}-\frac{\delta z_{g}}{8 f_{0}^{2} R^{2}} r^{4}+\frac{\delta z_{g}\left(R^{2}+8 f_{0} \delta z_{g}\right)}{96 f_{0}^{4} R^{4}} r^{6}+\mathcal{O}\left(r^{8}\right) .
$$

The former ray approximation approach can be generalized to the off-axis case, where the mirror has no more rotational symmetry. Thus, two spatial coordinates are required, as illustrated in Fig. 1(b). The focal distance $f(r)$ is defined with respect to the optical axis $z$ (after reflection), while the radial coordinate $r$ is defined for the incoming beam with respect to the optical axis $z^{\prime}$ (before reflection, where the incoming beam has rotational symmetry). To obtain the sag $s(x, y)$ for given off-axis angle and focal dependence $f(r)$, we perform a numerical optimization with the Zemax software. The incoming beam is split, before reflection, into a set of beamlets having a uniform spatial distribution over the incident beam aperture. The rays located in a narrow ring with radius $r$ are targeted to focus on a point $f(r)$ along the optical axis $z$, according to Eq. (3). Then, we write a merit function based on the summed proximity of all the rays to their defined target points. Using the local optimization tool of the Zemax software, we find the surface sag $s(x, y)$, which minimizes the merit function. We verify the validity of our numerical approach by comparing it with the analytical solution obtained in the on-axis case [Eq. (4)] for the specific choice of $f_{0}, \delta z_{g}$, and $R$ presented in the following paragraph.

For the design, we use the following parameters: nominal focus is $f_{0}=185 \mathrm{~mm}$, a focal line parameter is $\delta z_{g}=$ $30 \mathrm{~mm}$, and off-axis angle is $12^{\circ}$. The design is performed for an incident beam with radius $R=27.5 \mathrm{~mm}$ and a uniform intensity profile. Using the previously mentioned optimization procedure, we obtain a surface $s(x, y)$ as a series of twodimensional polynomial expansion.

In general, on-axis and off-axis surfaces can be decomposed in Zernike polynomials [20]:

$$
s(r)=\sum_{m, n} a_{n}^{m} Z_{n}^{m}(\rho, \phi), \quad s(x, y)=\sum_{m, n} a_{n}^{m} Z_{n}^{m}(\rho, \phi),
$$

where $Z_{n}^{m}(\rho, \phi)$ are Zernike polynomials using Noll's notation [21], $\rho=r / R$ is the normalized radial coordinate, $\phi$ is the azimuthal angle, and the $a_{n}^{m}$ are the expansion coefficients. In Table 1 we summarize the low order coefficients $a_{n}^{m}$ for the specific parameters of the axiparabola presented before. The decomposition is performed for the on-axis analytical (third column), on-axis (forth column), and off-axis (fifth column) numerical optimization cases. We can see that the coefficients (excluding tilt) remain close to each other in all the cases. The off-axis case is different by a tilt term, which corresponds to the $12^{\circ}$ off-axis angle. All other asymmetric terms, which are not shown in the table, remain negligible after optimization.

It should be noticed that the optimized off-axis surface did not focus all the rays precisely to their targets, as opposed to the on-axis case, which has an exact solution. The focal line of the off-axis axiparabola appears to be curved. Moreover, the smaller the nominal focus and the larger the off-axis angle, the larger the curvature is.

The axiparabola was manufactured as a $76.2 \mathrm{~mm}$ diameter aluminum mirror with diamond turning machining. The tolerance for the surface irregularity was below $500 \mathrm{~nm}$ peak to valley and for the roughness was below $10 \mathrm{~nm}$ root mean squared. For the purposes of the guiding experiment, a hole with a radius of $7 \mathrm{~mm}$ was drilled at the center of the axiparabola. Finally, the surface of the axiparabola was coated with protected silver.

We used this axiparabola with the $0.85 \mathrm{~J}, 30 \mathrm{fs}$ laser pulses from the Salle Jaune laser facility which operates at a central wavelength of $800 \mathrm{~nm}$, spectral width full-width-at-halfmaximum (FWHM) of $50 \mathrm{~nm}$, and at a repetition rate of $1 \mathrm{~Hz}$. The beam had a quasi top-hat energy distribution and a diameter of $47 \mathrm{~mm}$. In order to measure the beam profiles along the focal line, the beam was first attenuated down to $50 \mathrm{~nJ}$ and imaged by a microscope objective onto a CCD camera. Different positions along the focal line were registered by translating the imaging system along the optical axis. The peak intensity at full energy was estimated by measuring the laser energy just before the axiparabola $(0.85 \mathrm{~J})$ and assuming no temporal spread of the pulse.

Table 1. Zernike Coefficients of the Axiparabola Sag for the On-Axis and Off-Axis Cases and Specific Parameters Presented in the Text

\begin{tabular}{lcccc}
\hline $\boldsymbol{a}_{n}^{\boldsymbol{m}}$ & Classical Name & $\begin{array}{c}\text { On-Axis } \\
\text { Analytical } \\
(\boldsymbol{\mu} \mathbf{m})\end{array}$ & $\begin{array}{c}\text { On-Axis } \\
\text { Numerical } \\
(\boldsymbol{\mu} \mathbf{m})\end{array}$ & $\begin{array}{c}\text { Off-Axis } \\
\text { Numerical } \\
(\boldsymbol{\mu} \mathbf{m})\end{array}$ \\
\hline$a_{0}^{0}$ & Piston & 0.486 & $0.486^{a}$ & $0.486^{a}$ \\
$a_{1}^{-1}$ & Tilt & 0 & 0 & 1445 \\
$a_{2}^{0}$ & Defocus & 273 & 275 & 278 \\
$a_{4}^{0}$ & Spherical & -5.16 & -5.08 & -5.14 \\
$a_{6}^{0}$ & 2nd spherical & 0.173 & 0.099 & 0.100 \\
\hline
\end{tabular}

${ }^{a}$ This coefficient was fixed to the analytical solution value to allow convergence. 
The experimental results were compared to the results of a Zemax diffraction simulation. For this simulation, we used polychromatic point spread function (PSF) calculation using the fast Fourier transform (FFT) method. Essentially, each simulated focal spot is represented by an incoherent weighted sum of PSFs for different spectral components of our laser. We used the quasi top-hat illumination beam shown in the inset in Fig. 2(b).

As can be seen from the experimental and simulation results depicted in Fig. 2(a), the axiparabola generated an almost uniform focal line with a length of about $20 \mathrm{~mm}$ and with a peak intensity that exceeds $4 \cdot 10^{17} \mathrm{~W} \mathrm{~cm}^{-2}$. As anticipated, the axiparabola focal depth is smaller than the design parameter $\delta z_{g}=30 \mathrm{~mm}$, since the illumination beam has smaller spatial size than ideal top-hat, which was used in the design. Nevertheless, the axiparabola focal depth is larger nearly by a factor of 200 than the focal depth of an ideal parabola with the same illumination beam and focal length, while having comparable lateral spot size. Note that the peak intensity is reduced by the same factor as the length of the focal line is increased. Compared to the diffraction simulation, the experimental peak intensity appears reduced by about $50 \%$ and no oscillation of the intensity is observed along the focal line. The lower intensity in the experiment can be traced back to wavefront aberrations coming from both the incident beam and the surface of the axiparabola. The lack of oscillations in the experimental case can be explained by a low sampling of the curve. Peak intensity drop at the end of the focal line could be explained as follows: focal spots that lie close to the end of the focal line are generated from a thin annuli of large aperture; therefore, they are more sensitive to aberrations and have a lower peak intensity. From the simulated focal line depicted in Fig. 2(b), we can see that it is tilted horizontally, reducing the off-axis angle by $0.4 \mathrm{mrad}$. It is also curved, having height of the arc less than $2 \mu \mathrm{m}$.

Several simulated and experimental spatial profiles along the focal line are shown in Fig. 3, first and second rows, respectively. The beam transverse size remains below $7 \mu \mathrm{m}$ FWHM over the $20 \mathrm{~mm}$ of the focal line. The smallest focal spot size is about $4 \mu \mathrm{m}$; it is obtained close to the end of the focal line, since it is generated from the large aperture part. As anticipated, the second row in Fig. 3 shows that in the experiment, the beam profile is aberrated. Nevertheless, the size of the central spot is similar in the experiment and the simulation. Since the spot size changes along the focal line and the peak intensity is nearly constant, the energy deposition in the spot along the focal depth is not uniform. If needed, this non-uniformity can be removed by choosing a modified $f(r)$ in Eq. (3).
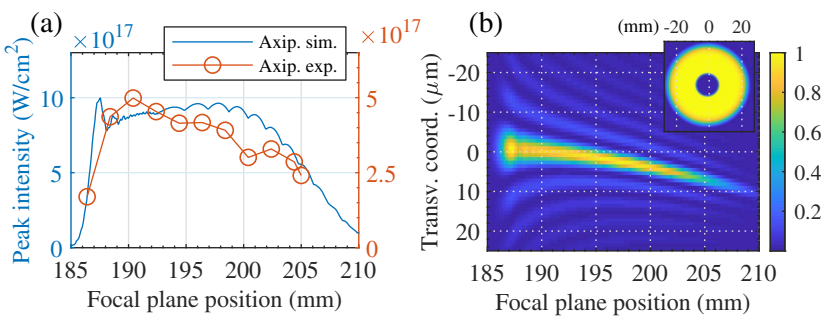

Fig. 2. (a) Axiparabola peak intensity as a function of the focal distance: red circles are the experimental data and the blue line comes from a polychromatic PSF simulation for a $0.85 \mathrm{~J}, 30 \mathrm{fs}$ pulse. (b) Simulated time integrated axiparabola beam profile cross section as function of the focal distance. Inset: Illumination profile used in simulation.

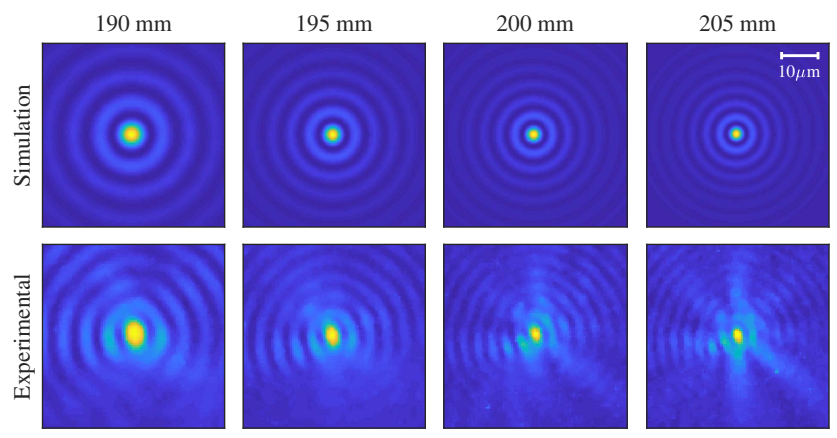

Fig. 3. Normalized beam profiles (for each plot) for different focal plane distances. The first row corresponds to simulated axiparabola focal spots. The second row depicts the experimental focal spots measured along the focal line of the axiparabola. The four columns correspond to the different focal plane distances: $190 \mathrm{~mm}, 195 \mathrm{~mm}$, $200 \mathrm{~mm}$, and $205 \mathrm{~mm}$, respectively. The colormap is the same as used in Fig. 2(b), while maximal value of each plot is 1 .

The capacity of axiparabolas to produce long focal lines with a narrow transverse size and high peak intensity makes them perfect tools for generating a plasma waveguide $[4,13]$. This plasma device is obtained by focusing in line a laser pulse in a low- $Z$ gas. At sufficiently high laser intensities $\left(I \gtrsim 10^{13} \mathrm{~W} \mathrm{~cm}^{-2}\right)$, the gas is ionized along the focal line, resulting in the formation of a plasma column. The latter then expands, leading after a delay of a few ps to a few ns (depending on the plasma temperature) to the generation of a plasma channel. It has a parabolic electronic density profile with the minimum at its center. This plasma channel can then act as a waveguide for another laser pulse. While the production of such a plasma column relies generally on collisional heating, because of the relatively low laser intensity attainable with axicons, here the gas can be directly ionized through optical field ionization. The main advantage of this ionization process is that its efficiency does not depend on the gas density; the use of axiparabolas may thus be a solution for application requiring a low plasma density, such as laser-plasma acceleration [13].

For demonstrating this potential, the two $30 \mathrm{fs}$ beams from the Salle Jaune laser facility were used. The simplified setup is shown in Fig. 4(a). A $0.85 \mathrm{~J}$ "generation pulse" (red color) was focused by the axiparabola on a $10 \mathrm{~mm}$ long hydrogen gas jet. The nozzle was located under the focal line positions ranging from 190-200 mm (Fig. 2). The unperturbed gas density was of $1 \times 10^{19} \mathrm{~cm}^{-3}$. After a duration of $3 \mathrm{~ns}$, a $0.6 \mathrm{~J}$ "guided pulse" (yellow color) was focused by a $1 \mathrm{~m}$ focal length parabola (f/18) at the entrance of the pre-formed plasma channel. A set of two glass wedges and neutral density filters was used to attenuate the guided pulse at the exit of the plasma. The focal spot was then imaged with an optical system comprising a singlet lens $(f=750 \mathrm{~mm})$, doublet lens $(f=450 \mathrm{~mm})$, microscope objective, and CCD camera. Translation of the singlet lens along the optical axis allowed to measure the guided pulse intensity profile in different planes.

Focal spots at the beginning of the gas jet, and at its end with no gas, with gas but without preformed channel, and with channel are shown in Figs. 4(b)-4(e). Because of spatial aberrations, the focal spot at the entrance has a low quality with a large part of the energy out of the central spot [Fig. 4(b)]. 


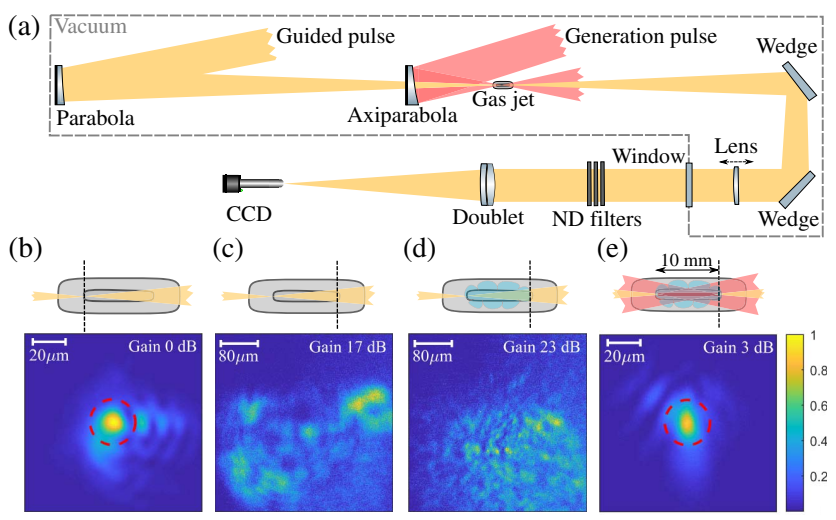

Fig. 4. (a) Experimental setup of the plasma waveguide experiment. A "generation pulse" is focused by an axiparabola on a hydrogen cloud above a $10 \mathrm{~mm}$ long gas jet. A "guided pulse" is then focused at the entrance of the pre-formed plasma channel. The output of the guided pulse is attenuated with two wedges and a set of neutral density (ND) filters. Different planes along the gas jet are imaged on a CCD camera. (b) Guided pulse spot at the entrance of the gas jet (no gas). (c) Guided pulse spot at the exit of the $10 \mathrm{~mm}$ gas nozzle without the plasma channel (no gas). (d) Guided pulse spot at the exit of the nozzle with the plasma. (e) Guided pulse spot at the exit of the nozzle with the preformed plasma channel.

Without the waveguide, the beam has significantly diverged in vacuum when it reaches the exit of the gas jet showing $17 \mathrm{~dB}$ of peak intensity reduction [Fig. 4(c)]. In Fig. 4(d), we can see that with gas, the beam has significantly diverged and the intensity is even more reduced than that in 4(c) due to scattering and depletion in the plasma. In contrast with the preformed waveguide, a small focal spot is obtained at the exit, which demonstrates the guiding of the 20 TW beam [Fig. 4(e)]. However, only $47 \%$ of the energy contained in the central spot (marked with dashed red circles in Fig. 4) is coupled into the plasma channel and guided to the exit of the plasma. The coupling efficiency and spatial profile of the guided beam at the exit of the plasma waveguide may be improved by reducing the wavefront aberrations of both the generation and guided beams and by using a gas medium with a more uniform density profile (such as a gas cell).

In conclusion, we presented a novel aspheric mirror (the axiparabola) enabling to generate long-focal-depth, high-lateralresolution beams. We derived a power series analytical solution for the on-axis case and proposed a numerical optimization for the off-axis case. A $20 \mathrm{~mm}$ focal depth, off-axis axiparabola was manufactured. We used it to produce a focal line with a transverse size and intensity that remained below $7 \mu \mathrm{m}$ and beyond $4 \times 10^{17} \mathrm{~W} \mathrm{~cm}^{-2}$, respectively. Achievable peak intensity is limited by the damage threshold of the mirror and could be increased above $1 \times 10^{18} \mathrm{~W} \mathrm{~cm}^{-2}$ by using $2.5 \mathrm{~J}$ pulses. This intensity paves the way to the use of line focus in the relativistic regime. As a first example of application, we used the axiparabola to generate a $10 \mathrm{~mm}$ plasma channel and guide a $20 \mathrm{TW}$ laser over nearly 10 Rayleigh lengths.

Axiparabolas present a large potential for tuning and optimization. Due to the use of diamond turning machining, the surface of the axiparabola can be shaped to generate focal lines with complex intensity profiles [solving Eq. (2) for a complex function $f(r)$ ]. To improve the coupling efficiency in guiding experiments, we may, for instance, design an optics that leads to larger intensity, and hence to a larger channel diameter at the beginning of the line. Besides optimizations for particular applications, a supplementary tuning can be achieved by coupling the axiparabola with a deformable mirror. The length of the focal line can, for instance, be changed by adding some spherical aberration to the incident laser beam. As a last example of their versatility, axiparabolas may also be used to generate highintensity, long hollow lines by adding a vortex phase either directly to the sag of the mirror or using additional optics.

Funding. Agence Nationale de la Recherche (ANR) (ANR13-BS04-0011) (LUCEL-X); Horizon 2020 Framework Programme (H2020) (730871); H2020 European Research Council (ERC) (339128); French Embassy in Israel (Chateaubriand fellowship).

Acknowledgment. We thank Dr. Dmitry Reshidko for assistance with Zemax.

\section{REFERENCES}

1. R. Meyer, M. Jacquot, R. Giust, J. Safioui, L. Rapp, L. Furfaro, P.-A. Lacourt, J. M. Dudley, and F. Courvoisier, Opt. Lett. 42, 4307 (2017).

2. S. Ahlawat, R. S. Verma, R. Dasgupta, and P. K. Gupta, Appl. Opt. 50, 1933 (2011).

3. Z. Ding, H. Ren, Y. Zhao, J. S. Nelson, and Z. Chen, Opt. Lett. 27, 243 (2002).

4. C. G. Durfee and H. M. Milchberg, Phys. Rev. Lett. 71, 2409 (1993).

5. J. H. McLeod, J. Opt. Soc. Am. 44, 592 (1954).

6. J. Durnin, J. Opt. Soc. Am. A 4, 651 (1987).

7. N. Davidson, A. A. Friesem, and E. Hasman, Opt. Lett. 16, 523 (1991).

8. J. Sochacki, Z. Jaroszewicz, A. Kołodziejczyk, and S. Bará, Opt. Lett. 17, 7 (1992)

9. J. Sochacki, A. Kołodziejczyk, Z. Jaroszewicz, and S. Bará, Appl. Opt. 31, 5326 (1992).

10. Z. Jaroszewicz, A. Kołodziejczyk, L. R. Staronski, and J. Sochacki, Opt. Lett. 18, 1893 (1993).

11. B.-Z. Dong, O. K. Ersoy, G.-Z. Yang, and B.-Y. Gu, J. Opt. Soc. Am. A 13, 97 (1996).

12. B. Wattellier, C. Sauteret, J.-C. Chanteloup, and A. Migus, Opt. Lett. 27, 213 (2002).

13. R. J. Shalloo, C. Arran, L. Corner, J. Holloway, J. Jonnerby, R. Walczak, H. M. Milchberg, and S. M. Hooker, Phys. Rev. E 97, 053203 (2018).

14. E. Esarey, C. B. Schroeder, and W. P. Leemans, Rev. Mod. Phys. 81, 1229 (2009).

15. M. Nisoli, S. D. Silvestri, O. Svelto, R. Szipöcs, K. Ferencz, C. Spielmann, S. Sartania, and F. Krausz, Opt. Lett. 22, 522 (1997).

16. A. Couairon and A. Mysyrowicz, Phys. Rep. 441, 47 (2007).

17. C. D'Amico, A. Houard, M. Franco, B. Prade, A. Mysyrowicz, A. Couairon, and V. T. Tikhonchuk, Phys. Rev. Lett. 98, 235002 (2007)

18. J. Kasparian, R. Ackermann, Y.-B. André, G. Méchain, G. Méjean, B. Prade, P. Rohwetter, E. Salmon, K. Stelmaszczyk, J. Yu, A. Mysyrowicz, R. Sauerbrey, L. Woeste, and J.-P. Wolf, J. Eur. Opt. Soc. 3, 08035 (2008).

19. J. Papeer, I. Dey, M. Botton, Z. Henis, A. D. Lad, M. Shaikh, D. Sarkar, K. Jana, S. Tata, S. L. Roy, Y. M. Ved, G. R. Kumar, and A. Zigler, Sci. Rep. 9, 407 (2019).

20. V. Lakshminarayanan and A. Fleck, J. Mod. Opt. 58, 545 (2011).

21. R. J. Noll, J. Opt. Soc. Am. 66, 207 (1976). 